\title{
LA CLASSIFICATION DE LA PARANOÏA DANS LA PSYCHIATRIE AMERICAINE CONTEMPORAINE : UNE REVUE DE LA LITTERATURE
}

\author{
Cécile Prudent, Renaud Evrard et Claude de Tychey
}

\section{Introduction}

Les entités nosographiques sont à la fois des productions scientifiques, et des objets pouvant être appréhendés historiquement et culturellement. La construction de ces entités reflète des enjeux qui vont bien au-delà d'un progrès linéaire des connaissances (1). C'est ainsi que les différentes versions du Manuel diagnostique et statistique des troubles mentaux (DSM), publié par l'American Psychiatric Association, ont fait l'objet de nombreuses études (par exemple, $(2,3,4))$. Dans une approche plus culturelle, c'est le contexte de la psychiatrie américaine qui a été examiné (par exemple, 5,6) ou l'exportation mondiale de cette culture singulière de la maladie (7). Jean Garrabé (8) concluait son analyse des nosographies et classifications dans l'histoire de la psychiatrie en affirmant que la validité de tels catalogues était limitée principalement à leur contexte culturel et historique de production, ce qui vient fortement questionner l'hégémonie actuelle de la psychiatrie américaine. Il ressort de ces études l'impression qu'au-delà de sa logique empirique et rationnelle, une classification à portée internationale telle que le DSM est aussi au carrefour d'enjeux extra-scientifiques. Certains ont pu être identifiés, mais d'autres nécessitent encore une prise de champ plus grande, ce que peut permettre une revue ciblée de la littérature.

L'entité nosographique à laquelle nous proposons de nous intéresser est la paranoïa. Diagnostic parmi les plus classiques en Europe, il est l'héritier de la primäre Verrücktheit de Wilhelm Sander (9) et de la monomanie de Ludwig Snell (10). En 1888, Richard von KrafftEbing (11) emploie le nom de paranoïa et bientôt Emil Kraepelin va lui donner une définition plus restrictive (12). Mais, comme le montre le travail de Benjamin Lévy (13), la paranoïa a fait office de «recapteur nosographique » entre 1865 et 1899 , c'est-à-dire d'entité dont l'existence semblait absolument nécessaire à la bonne marche du système psychiatrique car elle permettait de contenir un ensemble de cas indistincts. Cette notion de «recapteur nosographique " est toujours pertinente en ce qu'elle suggère une piste pour comprendre la situation actuelle, marquée par une disparition de la paranoïa dans le DSM-5, disparition qui entraîne une cascade d'effets sur les autres entités susceptibles d'absorber ce « reste » clinique - nous étudierons principalement le spectre de la schizophrénie - et sur les dispositifs de prise en charge. Il se pourrait ainsi que la paranoïa, devenue une notion trop stigmatisante et surtout trop catégorielle, ne soit plus apte à capturer les «phénomènes » qu'elle décrit. Allen Frances (4) voit dans ces évolutions nosographiques des «effets de mode », liés à des enjeux financiers, mais ceux-ci s'arriment sur des logiques intrinsèques qu'il est nécessaire d'évaluer de façon critique. L'évanouissement nosographique de la paranoïa est en fait un parfait exemple des répercussions, au sein de la psychopathologie anglo-saxonne, de la montée en puissance des approches dimensionnelles dans un paradigme post-psychanalytique (14). Plusieurs indicateurs montrent comment certaines entités sont en train de basculer d'un modèle auparavant purement catégoriel à des formes plus dimensionnelles, c'est-à-dire à une psychiatrie qui ne s'applique pas à la maladie mentale mais à la santé mentale, et qui explore 
les prémisses et les formes atténuées des psychopathologies le long de plusieurs continuums (15). À partir des éléments recueillis dans la littérature, nous tenterons de dégager une ou plusieurs hypothèses qui permettraient d'expliquer l'essoufflement de cette entité, et ce, malgré «sa longue histoire dans le lexique clinique » (16, p. 584).

\section{Histoire de l'intégration de la paranoïa dans la classification américaine}

La difficulté d'emblée rencontrée en anglais pour appréhender l'entité paranoïaque porte sur le fait qu'on ne dispose que d'un adjectif pour la désigner : "paranoid » (le plus souvent associé à des troubles ou à la schizophrénie), tandis que, de manière plus riche, la langue française permet de distinguer la «paranoïa», qui est un nom (une entité, unitaire et autonome, le nom désigne un être ou une chose), de «paranoïde » qui est un adjectif (mot qui qualifie le nom et qui s'accorde avec lui en genre et en nombre, le plus souvent associé à la schizophrénie dans la langue française). De sorte que l'anglais «paranoid »s'apparente plus à "paranoïaque » qu'à "paranoïde », semant de nombreuses confusions. Nous tâcherons, eu égard aux traductions officielles, de traduire par «paranoïde » ce qui ne fait que renvoyer à la dimension de persécution et par «paranoïaque » ce qui suggère une singularité clinique, tout en gardant à l'esprit que, de façon absolument problématique, ces distinctions ne sont pas faites par les auteurs de langue anglaise.

Dans son modèle de la «psychiatrie clinique », Kraepelin (17) a démontré l'importance de distinguer paranoïa et forme paranoïde (de la démence précoce). Si le délire paranoïde et le délire paranoïaque ont en commun la persécution, c'était dans le niveau d'élaboration du délire ou dans «l'affaiblissement mental » qu'il voyait résider des indicateurs diagnostiques différentiels. Dans la démence précoce, le délire paranoïde s'installerait sous forme «d'idées subites », « extravagantes », « incohérentes », « souvent régies par diverses hallucinations sensorielles ». Le délire paranoïaque au contraire se grefferait sur des bases qui ont «une existence réelle » mais dont le sujet déforme l'interprétation (17).

À la même époque Paul Sérieux et Joseph Capgras (18) définissaient le «délire d'interprétation », relatif à la paranoïa, comme un délire «systématisé » et «chronique ». Mais Eugène Bleuler (19) a ensuite influencé la pensée clinique en inscrivant le trouble délirant paranoïaque en tant que forme de schizophrénie (avec les paraphrénies et la catatonie). Subséquemment, deux courants divergents ont émergé dans la nosologie des troubles psychotiques paranoïdes : l'un étendant la perspective de Bleuler en intégrant les psychoses paranoïdes au groupe des schizophrénies ; l'autre comme extension de la vision de Kraepelin avec une paranoïa considérée indépendamment de la démence précoce et où :

«le délire "bizarre" est absent, il est organisé en un système logique et cohérent, les troubles de la pensée sont absents et la détérioration de la pensée systématiquement absente. Cette affection ne s'orientera généralement pas vers une "démence précoce". » (17, p. 204)

L'objectif du présent article est de mettre en lumière ce débat nosographique qui est toujours d'actualité. De notre place, inspirés par la théorie psychanalytique et psychodynamique européenne, la disparition de cette entité psychopathologique dans les classifications nous semble dommageable. Mais quelle est la position des chercheurs et des cliniciens à un niveau international ? Et pourquoi assistons-nous à cette disparition progressive ?

Nous tenterons de répondre à ces questions en retraçant brièvement l'histoire de la paranoïa au sein de la nosographie américaine durant ces dernières décennies dans le premier axe de ce travail. Dans un second axe, nous recenserons les différentes études, pour la plupart 
quantitatives, qui tendent à valider ou à infirmer le modèle psychiatrique américain. Dans un troisième axe, enfin, nous traiterons la question des échos de cette disparition sur l'entité voisine de schizophrénie.

\subsection{Du DSM I au DSM-5}

Les deux premiers opus du DSM, dans l'immédiat après-guerre, sont encore fortement marqués par la nosographie psychanalytique et par l'influence de la tradition clinique européenne (3).

Nous observons une double tendance, née dans les années 1980 avec le DSM-III (20), à faire entrer, tout en les distinguant, les entités PPD («paranoid disorder») et DD («delusional disorder ») dans la sphère des schizophrénies.

Dans le DSM-IV (20), ce schéma est maintenu.

Dans le DSM-IV-TR (21), le vaste spectre des «troubles paranoïaques » est divisé en trois segments et relogé à trois enseignes : son noyau se situe du côté de la « schizophrénie paranoïde (paranoid schizophrenia) ». Autour de ce noyau de schizophrénie gravitent, par ordre d'éloignement, les «troubles délirants (delusional disorders)» puis la «personnalité paranoïaque (paranoid personnality) ».

Nous assistons maintenant à une exclusion progressive du terme de la «paranoïa » au sein de la nosographie psychiatrique, puisque les «troubles de la personnalité paranoïaque » (Paranoid Personality Disorders, PPD), encore présents dans le DSM-IV TR, ont été supprimés du nouveau modèle du DSM-5 (22) et que, comme par glissement, les «troubles délirants » (Delusional Disorders, DD) apparaissent au sein du spectre schizophrénique. Nous nous attacherons à développer cette question de l'évolution de l'entité paranoïaque au sein de la nosographie psychiatrique anglo-saxonne jusqu'à son " évanouissement nosographique ». La disparition progressive des entités associées au terme «paranoïa » dans les différentes versions du DSM est visible dans le tableau 1, établi par Lévy (23). 
Tableau 1 : Entités associées au terme «paranoïa » au fil des versions successives du DSM

\begin{tabular}{|c|c|c|}
\hline Version & Terme anglais & aise \\
\hline $\begin{array}{c}\text { DSM-I, } \\
1952\end{array}$ & $\begin{array}{cc}- & \text { PARANOIA (systematized delusion) } \\
\text { - } & \text { PARANOID STATE (unsystematized } \\
\text { delusion) } \\
\text { - } \\
\text { - } \quad \text { PARANOID REACTIONS } \\
\text { (persecutory or grandiose) } \\
\text { - } \quad \text { INVOLURTIONONIC REACTION, } \\
\text { PARANOID TYPE } \\
\text { PARANOID TYPE } \\
\end{array}$ & 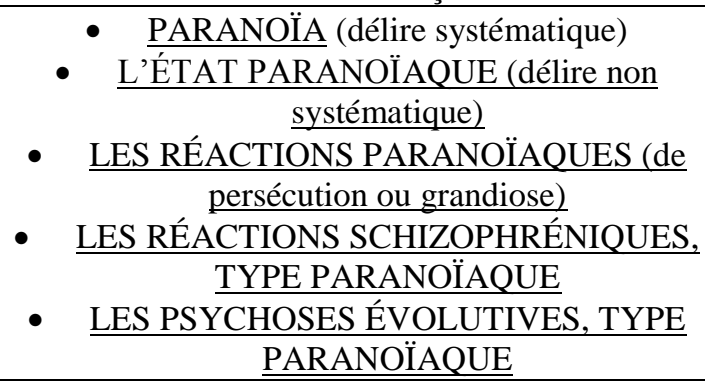 \\
\hline$\frac{\text { DSM-II, }}{\underline{1968}}$ & 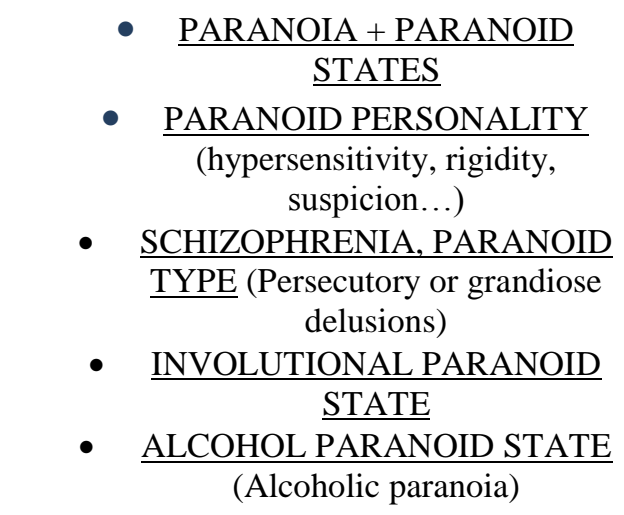 & $\begin{array}{c}\text { PARANOÏA + ÉTATS } \\
\text { PARANOÏAQUES } \\
\text { - } \quad \text { LA PERSONNALITÉ PARANOÏAQUE } \\
\text { (hypersensibilité, rigidité, suspicion... } \\
\bullet \quad \text { SCHIZOPHRÉNIE DE TYPE } \\
\text { PARANOÏDE (de persécution ou délires } \\
\text { de grandeurs) } \\
\text { - } \quad \text { L'ÉTAT PARANOÏAQUE ÉVOLUTIF } \\
\text { - ÉTAT PARANOÏAQUE INDUIT PAR } \\
\text { L'ALCOOL }\end{array}$ \\
\hline $\begin{array}{c}\frac{\text { DSM-III, }}{\frac{1980 \&}{D S M-I I I-R}} \\
\frac{1987}{\text { DSt }}\end{array}$ & 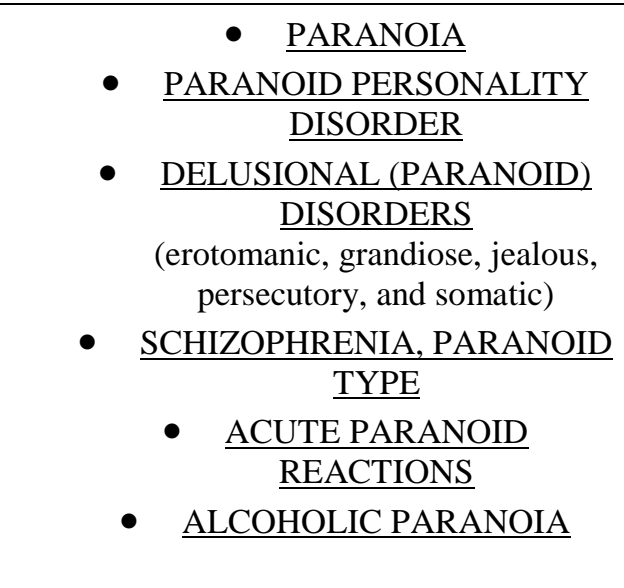 & 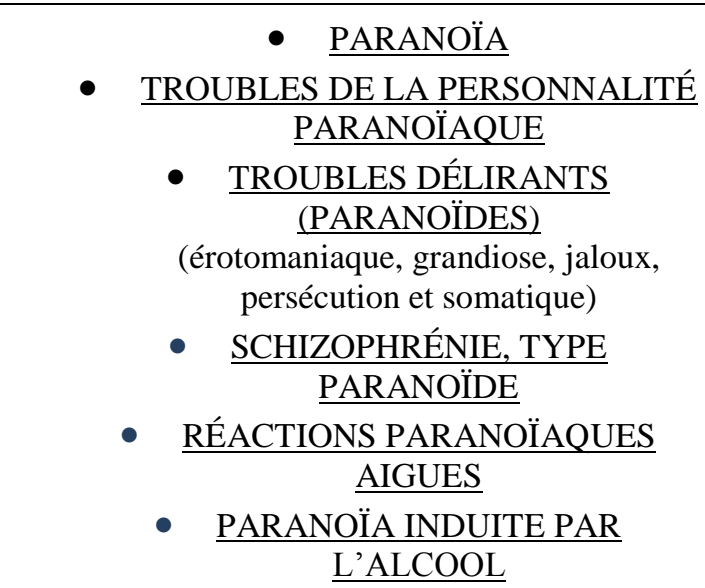 \\
\hline $\begin{array}{l}\frac{\text { DSM-IV, }}{1994 \&} \\
\underline{\text { DSM-IV- }} \\
\underline{\text { TR, 2002 }}\end{array}$ & $\begin{array}{cc}\text { - } & \frac{\text { SCHIZOPHRENIA, PARANOID }}{\text { TYPE }} \\
\text { - } & \frac{\text { PARANOID PERSONALITY }}{\text { DISORDER }} \\
\text { - } & \frac{\text { DELUSIONAL DISORDER }}{\text { (erotomanic, jealous, grandiose, }} \\
\text { persecutory, somatic and mixed subtypes) }\end{array}$ & $\begin{array}{c}\text { - SCHIZOPHRÉNIE, TYPE } \\
\text { PARANOÏDE } \\
\text { - TROUBLES DE LA PERSONNALITÉ } \\
\text { PARANOÏAQUE } \\
\text { - TROUBLES DÉLIRANTS } \\
\text { érotomaniaque, jaloux, grandiose, } \\
\text { persécuté, somatique et sous-types } \\
\text { mixtes) }\end{array}$ \\
\hline$\frac{\text { DSM-5, }}{\underline{2013}}$ & $\begin{array}{c}-\frac{\text { SCHIZOPHRENIA SPECTRUM }}{\frac{\text { AND OTHER PSYCHOTIC }}{\text { DISORDERS, (Delusional }}} \\
\text { - } \frac{\text { disorder) }}{\text { PERSONALITY DISORDER }} \\
\text { SParanoid Schizoid Schizotypal } \\
\rightarrow \text { Paranoid: suspicious, feels easily } \\
\text { rejected, tends to hold grudges. }\end{array}$ & $\begin{array}{lc}\text { - } & \frac{\text { SPECTRE SCHIZOPHRÉNIQUE ET }}{\text { AUTRES TROUBLES }} \\
\text { PSYCHOTIQUES (Trouble délirant) } \\
\text { - } \\
\begin{array}{c}\text { TROUBLES DE LA PERSONNALITÉ } \\
\text { (paranoïaque, schizoïde, schizotypique) } \\
\rightarrow \text { Paranoïaque : suspicion, se sent } \\
\text { facilement rejeté, tend à garder rancune. }\end{array}\end{array}$ \\
\hline
\end{tabular}




\subsection{La paranoỉa aux deux facettes}

La disparition de la paranoïa a été précédée par son démantèlement en deux facettes :

a) Le trouble de la personnalité paranoïaque («paranoid personality disorder », le PPD) qui correspond à la version plutôt interprétative de la pathologie paranoïaque ;

b) Le trouble délirant («delusional disorder», le DD) qui correspond à la symptomatologie délirante de la structure paranoïaque.

Comme 1'écrit Paul-Claude Racamier, entre ces deux facettes de la paranoïa, il pourrait n'y avoir qu'une différence de versant : «en fait il n'est pas de paranoïaque délirant qui n'ait un caractère perturbé, et pas de paranoïaque qui ne soit au bord de délirer ; caractère et délire font corps de façon très étroite » $(24$, p. 163). Ces deux facettes s'articuleraient comme variétés d'un même processus évolutif, ce qui n'empêcherait pas de constater des points d'oppositions, d'ailleurs relevés par le même auteur, soit que «le délire est plus manifeste alors que le caractère est plus insidieux », ou que «le délirant va en justice tandis que le caractère manœuvre dans l'ombre », ou encore qu' « il y a plus de masochisme chez le délirant et plus de haine chez le caractère ». Ces deux facettes sont également repérées et articulées dans le point de vue psychanalytique structural de Jean Bergeret (26), où elles correspondent à des niveaux de décompensation différents induits par la nature des mécanismes défensifs privilégiés par le sujet qui altère des niveaux différents du rapport à la réalité.

Quoi qu'il en soit, ces deux versants ont vu leurs destinés disjointes. Nous verrons comment, en incluant l'ensemble des délires dans les thématiques invraisemblables ou bizarres (sur la base des onze critères de Schneider, 27) du spectre de la schizophrénie, la nosographie américaine a eu tendance à faire disparaître la paranoïa. C'est ainsi que dans le DSM-5, le DD s'est dissolu en s'inscrivant dans le spectre schizophrénique, venant encore un peu plus marquer la confusion.

Concernant le trouble de la personnalité paranoïaque (le PPD), s'il existe encore dans la partie consacrée aux troubles de la personnalité (modèle classique) du DSM-5, il a disparu du modèle hybride qui est soumis depuis mai 2014 à la validation internationale en vue d'une adoption définitive. Cette modification tend à introduire une nouvelle perspective quasidimensionnelle des troubles psychotiques dans la nosographie psychiatrique américaine, en répartissant les signes de la psychose le long d'un continuum uniquement psychopathologique allant d'expressions bénignes à sévères d'un même "phénotype » de la psychose (28).

S'il est reconnu que l'introduction d'une perspective quasi-dimensionnelle (étalonnée en plusieurs niveaux) des troubles psychotiques était nécessaire étant donné que le modèle catégoriel (en terme de normal ou pathologique) n'était pas très bien adapté à la clinique (29), la disparition de l'entité " paranoïa », pourtant décrite depuis des décennies dans les manuels de psychiatrie, est néanmoins regrettable pour de nombreux cliniciens (par exemple : 30, 31). La paranoïa conserve également une place distincte de celle de la schizophrénie, au sein de la lignée psychotique, dans les approches structuralistes psychanalytiques, qu'elles soient bergerétienne ou lacanienne (15). Pour des praticiens ayant adopté ces référentiels, un tel démantèlement ravageur n'est pas sans évoquer ceux de l'hystérie et des névroses à partir du DSM-III (2).

Etant donné que les entités DD et PPD sont au centre du phénomène de disparition de la paranoïa dans les classifications américaines les plus récentes, nous les redéfinirons dès à présent à partir d'une revue de la littérature afin de mieux en comprendre leurs déterminants. Puis, afin de mettre en évidence les conséquences d'une évolution catégorielle vers une approche quasi-dimensionnelle, nous établirons une description contrastée de chacune d'elles. 


\section{Le trouble délirant, une entité complexe et polymorphe}

\subsection{Les troubles délirants (Delusional Disorders)}

Les troubles délirants (le DD) sont définis dans le DSM-IV (20) comme « la survenue d'une idée délirante unique ou d'un ensemble d'idées délirantes apparentées, et qui persiste habituellement toute la vie ». Le terme de troubles délirants est aussi utilisé dans la dixième édition de la Classification Internationale des Maladies (CIM-10, 32) dans un sens très proche de celui-ci.

Les principales critiques adressées à cette proposition classificatoire concernent sa complexité et son polymorphisme. Selon de nombreux auteurs $(32,33,34,35,36)$, cette difficulté a rendu le repérage diagnostique compliqué avec des répercussions néfastes sur la prise en charge des patients concernés ainsi que pour la recherche scientifique dans ce domaine. En effet, bien que ce concept fut défini d'une manière qui le distinguait de la schizophrénie, la description commode n'était «pas basée sur la compréhension des mécanismes qui pourraient provoquer les variations phénotypiques » comme l'affirment Christopher F. Fear, Tom McMonagle et David Healy en 1998 (37, p. 213). D'ailleurs, comme le relèvent ces auteurs, la catégorisation relativement vague et étendue des troubles délirants a fait l'objet d'un débat lors de sa classification internationale, tout comme elle avait déjà suscité de grandes controverses au début du siècle dernier en France et en Allemagne (cf.13). Le débat se situe toujours entre le spectre schizophrénique et les troubles représentatifs de la paranoïa. Force est donc de constater que cette entité n'a jamais vraiment pu trouver sa place au sein de la nosographie psychiatrique, ce qui est probablement lié à la base purement symptomatologique sur laquelle certains ont tenté de baser sa classification.

Plusieurs alternatives sont évoquées par nombre d'auteurs qui ont contribué à alimenter le débat sur la classification de la paranoïa dans le modèle psychiatrique américain. Par exemple, afin de mieux identifier le DD en tant qu'entité psychopathologique, il aurait été nécessaire, selon Fear et al. (37) de réaliser plusieurs classes au sein même de ce syndrome jugé bien trop hétérogène. Dans le même temps, Alistair Munro dans son ouvrage en 1999 (38), souligne, quant à lui, le caractère multidimensionnel, complexe et fluctuant de DD et critique explicitement cette classification qu'il estime trop « rigide ». L'auteur considère qu'il s'agit «d'un réductionnisme opacifiant» qui ne tient pas compte des soubassements psychopathologiques du trouble (DD). Il déplore notamment que les nombreux travaux des psychodynamiciens aient été considérés avec scepticisme par les auteurs du DSM, et attribue à leur position résolument «simpliste » la responsabilité d'une répétition des conflits déjà rencontrés dans l'histoire de la psychiatrie.

Dès lors, on assiste, à partir de la fin des années 1990, à une forme d'émulation réactionnelle productrice d'une suite de modèles :

- Munro (38) distingue, dès 1999, quatre sous-types au sein de DD : (i) le sous-type grandiose, (ii) le sous-type de persécution, (iii) le sous-type érotomaniaque, (iv) le sous-type mixte.

- Parallèlement, Alessandro Serretti, Enrico Lattuada, Cristina Cusin et Enrico Smeraldi (39) mettent en évidence, à partir de l'analyse factorielle qu'ils ont réalisée chez 52 sujets DD, une symptomatologie organisée en 4 facteurs : (i) la dépression, (ii) les hallucinations, (iii) le délire d'interprétation, (iv) les symptômes d'irritabilité.

Ces facteurs permettent, selon ces auteurs, de constituer un profil homogène des sujets souffrant de DD. Mais, selon nous, ces observations semblent plutôt correspondre au profil paranoïaque. En effet, Sigmund Freud (40) avait déjà mis en évidence ces éléments au sein de la paranoïa et notamment la valence dépressive chez Schreber (à travers ses tentatives de suicide), la dépression pourrait être comprise, dans cette structure, comme résultant de 
l'introjection de l'objet perdu (41); et l'irritabilité comme l'expression du mécanisme de défense connu chez les psychanalystes et psychodynamiciens sous le nom de projection ou d'identification projective ; les hallucinations et le délire relevant, selon Jacques Lacan (42), d'une projection dans le Réel de ce qui n'a pu être symbolisé, sont associées pour Jean Bergeret (26) à des mécanismes massifs de déni de la réalité, et, chez Freud (40), comme les derniers bastions avant le morcellement du Moi.

D'autres découpages ont été proposés :

- George Winokur (43) de l'université de l'Iowa, a quant à lui, décrit les principaux troubles cliniques des patients du DD comme étant constitués (i) d'un délire de référence, (ii) de jalousie, (iii) de persécution, (iv) de problèmes sexuels plutôt courants. Pour lui, l'hypocondrie et la grandeur sont rencontrées mais ne sont pas fréquentes. Ces données analysées par Winokur (43) peuvent être comprises comme les différentes expressions de la paranoïa qui n'a pas un mode unique d'expression en dépit du moule dans lequel voudrait la faire entrer les auteurs du DSM.

Ainsi, nombreux sont les auteurs qui reconnaissent l'hétérogénéité symptomatique des troubles délirants : de fait, le DSM-IV et le DSM-5 classifient ce trouble en fonction de sept sous-types d'idées délirantes (types érotomanie, grandiose, jaloux, de persécution, somatique, mixte et non spécifié). Enrique de Portugal et al. (44) déplorent cette hétérogénéité et considèrent que c'est une erreur de fonder le diagnostic de trouble délirant en se basant uniquement sur les thématiques délirantes, comme l'avait déjà critiqué Kraepelin à son époque. A ce sujet, il écrivait : "la nature du délire n'est que d'un faible secours pour l'aliéniste chargé de formuler le diagnostic d'un syndrome morbide » (17, p. 180).

\subsection{Troubles délirants et normativité du « bizarre »}

Sur un plan théorico-clinique, comme le font remarquer Fear et al. (37), nous ne pouvons qu'observer un appauvrissement dans la démarche diagnostique au sein du DSM-IV-R car il excluait du diagnostic de DD tout sujet qui présentait un «délire bizarre ». En effet, par ce biais, on obligeait quasi-systématiquement le clinicien à poser un diagnostic de schizophrénie. En incluant l'ensemble des délires dans les thématiques « invraisemblables » ou «bizarres », sur la base des critères de premier rang de Schneider (27), la nosographie américaine tend ainsi à englober de manière inéluctable l'ensemble des paraphrénies dans le spectre schizophrénique.

On peut néanmoins se demander quelle part prend la subjectivité et les influences socioculturelles dans cette détection de la bizarrerie. Le concept de bizarrerie (peculiarity en anglais) est décrit par plusieurs auteurs comme une variable multidimensionnelle déterminant des différences entre les individus (45). La bizarrerie implique un continuum où, à l'un des extrêmes, «les croyances bizarres sont considérées comme des délires, et les perceptions bizarres comme des hallucinations » (45, p. 27). Dans le DSM-IV-R, la qualification de bizarre est pourtant définie de façon quasi-objective :

«Les idées délirantes sont considérées comme bizarres si elles sont nettement invraisemblables et incompréhensibles et ne proviennent pas d'expériences ordinaires de la vie. Un exemple d'idée délirante bizarre est la croyance qu'un étranger a enlevé les organes internes du sujet et les a remplacés par ceux de quelqu'un d'autre sans laisser de plaies ou de cicatrices. Une idée délirante non bizarre est la croyance fausse qu'il ou elle est sous surveillance policière. » (22, p. 345-346)

D'autres recherches sont venues complexifier voire contredire la définition du DSM.

- Madeline M. Gladis, Douglas F. Levinson et Bryan J. Mowry (46) ont montré la difficulté à classifier des délires, ne trouvant pas de différences consensuelles entre les 
croyances étranges et les délires complets, les délires transitoires et les délires persistants, les délires bizarres et les délires non-bizarres.

- Ramin Mojtabai et Robert A. Nicholson (47) ajoutent que, même dans les cas de délires bizarres, supposés les plus faciles à classer, la fiabilité des cotations de 50 psychiatres réunis en cette occasion était très insatisfaisante.

En définitive, la notion de bizarrerie a été presque totalement abandonnée dans le DSM-5. Pour le psychiatre Alain Bottéro (48, p. 43), il est évident que cette qualification de bizarre laisse trop de latitude d'interprétation à la discrétion du psychiatre et ne peut être affaire que de normes intellectuelles bien délicates à appliquer de façon tranchée. Le psychanalyste JeanClaude Maleval (49, p. 226) invite, quant à lui, à prendre le contre-pied de cette position clinique : "Quelle que soit la bizarrerie d'une idée, elle ne saurait suffire à désigner un sujet comme délirant. Rien ne fait limite à l'imagination créatrice. »

Si bien que le diagnostic différentiel paranoïa/schizophrénie basé sur une normativité du bizarre ne rend pas justice à la complexité de cette clinique.

L'ambiguïté et la confusion que cela pouvait créer a été levée puisque le DD y est désormais situé dans le spectre schizophrénique. Le contenu bizarre du délire peut toujours fait l'objet d'une spécification diagnostique, mais n'a plus valeur de signe différentiel.

\subsection{Appréhension clinique des troubles délirants}

Comme nous pouvons le constater, l'appréhension des troubles délirants, introduits par l'APA en 1980 (20) ne fait aucunement l'objet d'un consensus au sein de la communauté scientifique internationale, tant au niveau de la forme que du fond. Les différents auteurs que nous venons de citer, spécialistes de la classification psychiatrique (sur une base symptomatologique), expriment la grande difficulté à intégrer cette entité dans le modèle classificatoire du DSM pour les raisons suivantes :

- Le DD est polymorphe, il ne circonscrit pas de façon optimale un trouble de la personnalité pourtant reconnu (la paranoïa);

- Le DD est complexe, la classification psychiatrique classique sur la base des troubles n'est pas adaptée à cette pathologie.

Une des principales conséquences, néfaste tant sur le terrain que dans le domaine de la recherche appliquée, est que la prévalence de cette pathologie est certainement sous-estimée. En effet, le trouble délirant est classiquement considéré comme peu fréquent ; il représente 1 à $2 \%$ des diagnostics selon Maâlej et al. (35). Kenneth S. Kendler, William M. Glazer et Jacob M. Morgenstern (50) estiment quant à eux qu'il y a 1 à 3 nouveaux cas par an de trouble délirant pour 100000 patients. Alexandre Madeira, Lucas Francisco Manarte, Rafael D. Guerreiro et Sara Dias (51) considèrent qu'il représente seulement entre 2 et $8 \%$ des hospitalisations psychiatriques.

En revanche, dans une étude finlandaise menée par Jaana M. Suvisaari et al. en 2008 (52), portant sur 8028 sujets finlandais diagnostiqués psychotiques selon le DSM-IV-TR (22), $18 \%$ des patients portent le diagnostic de DD et seulement $50 \%$ d'entre eux reçoivent un traitement.

Il existe donc une grande disparité dans la reconnaissance de ces troubles sur le terrain en fonction de la sensibilité des praticiens. Ce «manque à gagner » résulterait d'une forme de négligence dans ce domaine depuis de nombreuses années (38). C'est également le constat de Catherine Azoulay (53, p. 36) qui écrit :

«Si le terme même de paranoïa est entré dans le vocabulaire courant, l'intérêt des psychiatres, mais aussi de nombre de psychanalystes, s'est détourné, depuis un certain nombre d'années, de l'étude de ses aspects pathologiques au profit d'une centration sur les schizophrénies et des troubles dépressifs. » 
Il semble donc nécessaire de développer des stratégies diagnostiques adaptées à ce type de trouble.

\section{Le trouble de la personnalité paranoïaque (Paranoid Personality Disorder), une seconde entité représentative de la paranoïa classique dans la psychiatrie moderne}

Selon le DSM-5 (23), la caractéristique essentielle du trouble de la personnalité paranoïaque correspond à une méfiance généralisée et notamment en la méfiance des autres, de sorte que leurs motivations sont interprétées comme malveillantes. Bien qu'ils peuvent sembler être objectifs, rationnels, et sans émotion, ils affichent le plus souvent une gamme labile de l'affect, avec des expressions hostiles, tenaces, et sarcastiques prédominantes.

C'est dans des circonstances liées au stress que les personnes atteintes de ce trouble peuvent éprouver de très brefs épisodes psychotiques (durée estimée entre quelques minutes et quelques heures). Dans certains cas, le trouble de la personnalité paranoïaque peut apparaître comme l'antécédent prémorbide du trouble délirant ou de la schizophrénie.

Les personnes atteintes de trouble de la personnalité paranoïaque peuvent développer des accès dépressifs majeurs et sont enclins à l'agoraphobie et aux troubles obsessionnelcompulsifs. L'alcool et les autres troubles de dépendance (toxicomanie) sont également fréquents. Les facteurs de comorbidité surviennent plus couramment dans les troubles de la personnalité schizotypique, schizoïde, narcissique, d'évitement et borderline. (23)

La prévalence du PPD indiquée dans le DSM-5 est estimée sur la base d'un sous-échantillon de probabilité de l'étude nationale des comorbidités «National Comorbidity Survey Replication » qui suggère une prévalence de 2,3\%, tandis que l'enquête épidémiologique nationale sur l'alcool et les affections apparentées, citée également dans le DSM-5, obtient des données qui suggèrent une prévalence du trouble de la personnalité paranoïaque de $4,4 \%$. Ce qui signifie que la disparité entre les différents travaux est importante et qu'il n'y a actuellement pas de consensus sur les données épidémiologiques en lien avec le PPD.

Les risques et les facteurs pronostiques sont associés, selon les auteurs du DSM-5, aux troubles schizophréniques puisqu'il existe certaines preuves (23) mettant en évidence une prévalence accrue du trouble de la personnalité paranoïaque chez les sujets dont on retrouve chez les parents la présence d'un lien familial avec le trouble délirant et notamment le type de persécution.

[Insérer tableau 2]

Tableau 2 : Les troubles de la personnalité paranoïaque (DSM-5, APA, 2013, p. 649)

PARANOID PERSONALITY DISORDER

Critère diagnostic 301.0 (F 60.0)

A. Une méfiance généralisée ainsi que la méfiance des autres de sorte que leurs motivations sont interprétées comme malveillantes, commençant à l'âge adulte et présents tôt dans une variété de contextes, comme indiqué par quatre (ou plus) de ce qui suit:

1. Les suspicions sans fondement, comme celles que d'autres les exploitent, veulent les blesser, ou les tromper. 
2. sont préoccupés par des doutes injustifiés sur la loyauté ou la fiabilité des amis ou des associés.

3. sont réticents à se confier à d'autres en raison de la crainte injustifiée que l'information sera utilisée avec malveillance contre eux.

4. ont une lecture des événements (bénins), ou des remarques d'autrui, où la honte et l'humiliation sont cachées.

5. une forte persistance à la rancune (i.e. : sont impitoyables concernant les insultes, les blessures ou les affronts).

6. perçoivent des attaques sur leur caractère ou leur réputation qui ne sont pas évidentes pour les autres et sont prompts à réagir avec colère ou à contre-attaquer.

7. ont des soupçons récurrents, sans justification, en ce qui concerne la fidélité de leur conjoint ou de leur partenaire sexuel.

B. Ne se produit pas exclusivement au cours de la schizophrénie, d'un trouble bipolaire ou d'un trouble dépressif avec des caractéristiques psychotiques, ou d'un autre trouble psychotique et ne sont pas attribuable aux effets physiologiques d'une autre condition médicale.

Remarque: Si les critères sont remplis avant l'apparition de la schizophrénie, ajouter «prémorbide», à savoir, « paranoïaque trouble de la personnalité (prémorbide). »

\subsection{L'émergence de l'approche dimensionnelle}

La présence de traits de personnalité attenants aux troubles catégorisés dans la nosographie psychiatrique chez le sujet «non-clinique» est donc au fondement d'une approche dimensionnelle. On trouve déjà le débat des antagonismes catégoriels et dimensionnels dans les travaux de Carl Gustave Hempel (54) et d'Hans Eysenck (55) à la fin des années 1980. Hempel (54) proposa, en effet, de développer une approche pragmatique des troubles dans Fundamentals of Taxynomy en 1965. Dragan M. Svrakic, Cynthia Whitehead, Thomas R. Przybeck et C. Robert Cloninger (56), Lim Saulsman et Andrew C. Page (57), R. Mickael Bagby, Paul Costa, Thomas Widiger, Andrew G. Ryder et Margarita Marshall (58), Daneluzzo et al. (59), Freeman (60), Wilcox, Briones, Quadri et Tsuang (61) sont autant d'auteurs à avoir également repéré des traits de personnalité attenants aux troubles catégorisés dans la nosographie psychiatrique chez le sujet «non-clinique ».

Le caractère évolutif des troubles était déjà au centre de la conception kraepelinenne, comme le soulignent Magali Bricaud, Benjamin Calvet, François Viéban, Annie Prado-Jean, JeanPierre Clément. (62), qui rappellent que Kraepelin considérait que l'évolution d'une maladie caractérisait son individualité : «la folie n'est rien d'autre que l'exagération du caractère habituel » (12).

De même, Ersnt Kretschmer (63) utilisait les termes de «schizophrènes », «schizoïdie » et «schizothymie» pour décrire différentes graduations de la même disposition de tempérament: «c'est cette élaboration théorique inaugurée par Kretschmer qui donna 
naissance à l'approche dimensionnelle » (63, p. 302). Peu après, Carl Gustave Hempel (54) ayant jugé cette démarche féconde en psychopathologie proposera d'utiliser un schéma de classification en catégorie par un autre schéma «d'espace à plusieurs dimensions ». Bricaud et al. (62) se sont proposés, à travers une étude portant sur un large échantillon, de participer à ce débat nosologique :

«Il existe une corrélation entre certaines personnalités pathologiques du DSM-III-R et certaines dimensions tempéramentales, mais la puissance statistique est souvent modérée $»(62$, p. 29).

Selon ces auteurs, l'approche dimensionnelle pourrait venir compléter l'approche catégorielle dans la nosographie psychiatrique. C'est la proposition du DSM-5, modèle hybride où certains troubles de la personnalité sont maintenus selon une approche catégorielle et où le spectre schizophrénique absorbe le reste des psychoses, comme la paranoïa (dans les différents sous-types de schizophrénie), en s'inscrivant dans une approche dimensionnelle.

\subsection{La paranoïa-trait, une approche dimensionnelle favorisant la disparition de la paranoïa}

A partir des années 1990, certaines études $(56,57,58,59,64,60,61,66,67,68)$ ont observé une corrélation entre les différentes dimensions de la personnalité chez des sujets «sains » et les troubles de la personnalité proposés dans l'approche catégorielle du DSM. Ces données suggèrent que des symptômes de type paranoïaque ne sont pas rares dans les populations noncliniques, c'est-à-dire chez des individus n'ayant jamais consulté ou n'ayant jamais été diagnostiqués pour des troubles psychiatriques. Voici une brève description de certaines de ces études en provenance de populations variées dans différents pays :

- Dans une étude portant sur 4951 étudiants de premier cycle toutes disciplines confondues en Chine, Raymond C.-K. Chan et al. (65) repèrent une prévalence élevée d'«idées paranoïdes »: environ $18 \%$ des étudiants avaient fait l'expérience d'idéation paranoïde au moins une fois par semaine durant la période de l'étude ; $71,5 \%$ d'entre eux rapportaient avoir été en proie, au moins une fois dans leur vie, à un phénomène paranoïde ; et $56,3 \%$ déclaraient avoir souffert d'au moins deux symptômes relatifs à la paranoïa.

- Quelques années auparavant, Daniel Freeman et al. (60) avaient déjà relevé que 10 à $15 \%$ de la population générale faisait des expériences de pensées paranoïaques bien que seulement $0,7 \%$ à $3,7 \%$ des participants ne se prêtaient au diagnostic clinique de psychose.

- En 2012, Joseph H. Triebwasser, Erwin Chemerinsky, Panos Roussos et Larry J. Siever (67), dans une étude portant sur un échantillon de 914 sujets non hospitalisés et sans traitement médicamenteux, recrutés dans la région de New York, observent que $27 \%$ de ces individus répondent aux critères complets des troubles de la personnalité paranoïaque.

- Svenn Torgersen, Einar Kringlen et Victoria Cramer (66) ont mis en évidence une prévalence de 2,4\% à Oslo, en Norvège ; et Jeremy Coid et Min Yang (68) une prévalence pondérée de $0,7 \%$ au Royaume-Uni.

La lecture de ces travaux soulève une question de méthode car toutes ces données sont issues d'auto-questionnaires. Comment appréhender des idées paranoïdes sur la base d'autoquestionnaires qui ne permettent pas un retour et une évaluation critique sur la situation et le contexte? En plus des difficultés classiques liées aux bais d'auto-présentation et de désirabilité sociale que la communauté scientifique attribue aux auto-questionnaires en général, la plupart de ces tests ne comportent aucune échelle permettant d'apprécier le degré de cohérence et de sincérité du sujet qui répond. 
En France, un travail de recherche en cours, prélude à un doctorat, portant sur le diagnostic différentiel des psychoses (69) et utilisant conjointement le test de Rorschach et le MMPI2RF, va dans le même sens. Il suggère que les scores obtenus à l'échelle RC6 (idées de persécution) de ce dernier test ne permettent nullement d'identifier les sujets paranoïaques dont les réponses aux échelles de validité apparaissent, en outre, le plus souvent biaisées. En revanche, une épreuve projective telle que le test de Rorschach, administrée aux mêmes sujets, permet non seulement d'identifier ceux présentant un trouble de la personnalité paranoïaque, mais également de les différencier de leurs homologues schizophrènes à partir de marqueurs projectifs renvoyant au fonctionnement intrapsychique. Il semble que la méthodologie des tests projectifs pourrait avoir une pertinence clinique plus grande pour repérer les signes de la personnalité paranoïaque, comme l'ont souligné plusieurs cliniciens américains $(33,70)$.

Une interprétation de ces données a favorisé l'approche dimensionnelle de la personnalité paranoïaque au détriment d'une approche structurale. Les auteurs se référant aux approches structuralistes bergerétiennes ou lacaniennes voient dans la suppression de la paranoïa une erreur (68). Lacan s'était déjà opposé au délitement du type paranoïaque qui tendait, déjà à l'époque de sa thèse, à se confondre avec la notion de caractère (71). Pour Lacan (42), le terme de constitution paranoïaque se justifie « par la fixation précoce d'une structure ». Selon l'approche structurale lacanienne, il s'agirait de prendre en considération des oscillations entre des états ordinaires et des états extraordinaires de la structure, c'est-à-dire un déclenchement de manifestations cliniques qui serait ni inéluctable ni irréversible (49). Par un autre cheminement théorique, la perspective structurale défendue par Bergeret (26) repose sur l'idée d'un fonctionnement psychodynamique le long d'un continuum entre le normal et le pathologique, selon que la structure paranoïaque de base est compensée, instable ou décompensée, en fonction des conflictualités internes et externes qu'elle rencontre. Ces deux positions structuralistes intègrent les données suggérant une dimensionnalité tout en proposant un modèle mixte, avec une approche structurelle pour les entités psychotiques et névrotiques au sein desquelles les sous-entités schizophrénie, paranoïa, mélancolie/maniaco-dépression, obsessionnel et hystérie conservent leur autonomie (15). L'abandon de certains troubles de la personnalité spécifiques, des $\mathrm{DD}$ et des $\mathrm{PPD}$, et l'abord restreint de la paranoïa ramené uniquement à un trait de personnalité, conduisent inévitablement à la disparition progressive de la notion de structure paranoïaque.

Ces recherches multicentriques ont conduit les auteurs du DSM-5 à envisager la personnalité paranoïaque comme un trait de personnalité exclusivement, et non plus comme une constitution structurelle. Dès lors, la paranoïa-trait a semblé relever spécifiquement du spectre schizophrénique. Implicitement, les auteurs du DSM-5 renvoient l'idée que toute personne expérimentant des «pensées paranoïdes» serait potentiellement schizophrène dans une perspective quasi-dimensionnelle !

\section{Le débat sur la suppression de la paranoïa : trait paranoïaque ou entité autonome?}

Les troubles délirants (DD) et les troubles paranoïaques de la personnalité (PPD) sont en voie de disparition dans le DSM-5.

En réalité, selon David P. Bernstein (72, p. 536), il y a une «claire majorité des experts des troubles de la personnalité qui est insatisfaite du système diagnostique actuel ». Dans une autre étude, Mullins-Sweat, Bernstein et Widiger (31), ont montré que $80 \%$ des experts pensent que les troubles de la personnalité sont à comprendre comme des variances extrêmes de personnalité «normales » plutôt que des catégories d'entités distinctes. 
Ainsi, la proposition du DSM-5 qui va dans le sens d'une approche quasi-dimensionnelle, conforte ces chercheurs. Mais les experts du groupe de travail ont choisi de supprimer la moitié des troubles de la personnalité (dans le modèle hybride uniquement, ces entités restent maintenues pour l'instant dans le modèle classique du DSM-5). Parmi lesquels:

- la personnalité dépendante ;

- la personnalité histrionique ;

- la personnalité narcissique ;

- la personnalité paranoïaque ;

- la personnalité schizoïde ;

C'est ainsi que la paranoïa n'est plus considérée comme une entité autonome mais comme une variance extrême de la normale, comme un facteur de comorbidité au sein d'un des troubles de la personnalité, ou comme appartenant au spectre schizophrénique à travers le sous-groupe des schizophrénies paranoïdes.

\subsection{Un manque de recherches}

La place du trouble de la personnalité paranoïaque a été interrogée à plusieurs reprises, dans le cadre des révisions des systèmes diagnostiques DSM et CIM. Mais ces interrogations semblent s'appuyer sur peu de données nouvelles. Soren Fryd Birkeland (73) constate un «manque de données de recherches cliniques concernant le trouble de la personnalité paranoïaque ». (p.254) Selon Triebwasser, Chereminski, Roussos et Siever (67), «il y a relativement peu de données probantes publiées pour la fiabilité et la validité de troubles de la personnalité paranoïaque ».

Hopwood et Thomas (16) écrivent à propos des troubles de la personnalité paranoïaques et schizoïdes, qu'ils ne sont "pas déterminées sur la base des recherches existantes et que le complément d'enquête est nécessaire pour déterminer leurs statuts nosologiques ». Ces auteurs envisagent la possibilité que le DSM-IV-TR n'a pas évalué de manière adéquate la construction du PPD. Les limites de la recherche sur cette entité sont, selon eux, dues à la «mauvaise conceptualisation» de cette entité et non au construit en lui-même. Le PPD détient un certain potentiel, selon Hopwood et Thomas, il serait donc cliniquement et empiriquement important de continuer d'enquêter sur ses processus car actuellement ils considèrent que l'on assiste à une « négligence délibérée de l'étiologie et de la théorie ». (16, p. 595)

Pourtant, le trouble de la personnalité paranoïaque mériterait d'autant plus d'attention que c'est l'un des trois troubles de la personnalité les plus fortement associés à une réduction de la qualité de vie (66). Ceci pourrait être dû à la réticence des sujets paranoïaques à se soigner, qui elle-même expliquerait qu'on ne les rencontrerait pas tant en hôpitaux psychiatriques que dans des centres d'aide sociaux (ou en centre carcéral). C'est ce que confirmerait, par exemple, l'étude de Jennifer A. Connolly, Patricia Cobb-Richardson et Samuel A. Ball (74) qui ont mis en évidence que dans un échantillon de sujets issus d'un centre de sans-abri, le trouble de la personnalité paranoïaque était le plus répandu des troubles de la personnalité diagnostiqués. Cette tendance à refuser tout traitement et/ou de participer aux recherches (75) n'inciterait pas l'industrie pharmaceutique à investir des recherches auprès de cette population, ce qui renforcerait la pauvreté des travaux publiés.

D'ailleurs, on observe qu'il n'y a aucune étude menée en laboratoire publiée connue à ce jour qui étudie spécifiquement les troubles de la personnalité paranoïaque (67). Toutes les études ont été réalisées sur des échantillons qui incluent les patients atteints également d'autres troubles de la personnalité.

«Ainsi, les individus pour qui la paranoïa est une composante clé de leur problématique 
peuvent être précisément les personnes qui sont le moins enclines à s'engager dans la thérapie et à accepter une chimiothérapie tout comme ils ne sont pas faciles à recruter ou à conserver dans des essais de recherche. (...) à cause de leur utilisation de la projection, les patients qui souffrent de troubles de la personnalité paranoïaque peuvent être ceux qui sont le plus en difficulté de tous les patients psychiatriques pour localiser la source de leurs problèmes plutôt venant d'ailleurs que venant d'eux-mêmes. » (67, p.2).

Triebwasser et al., (67) supposent que le manque de travail de recherche sur la paranoïa relève $\mathrm{du}$ fait que ces sujets soient peu disposés à suivre un traitement psychothérapique ou chimiothérapique et qu'ils localisent la cause de leurs problèmes à l'extérieur et non en eux-mêmes. Cette tendance paranoïaque à éviter le soin et la recherche pourrait être de nature à expliquer le désintérêt des laboratoires pharmaceutiques pour cette entité. Or le traitement médicamenteux tient une place très importante dans le «nouveau paradigme de la psychiatrie », comme l'a soulevé Allen Frances (4). Il est possible que la paranoïa ait en partie disparue de la nosographie à cause du manque de soutien de l'industrie pharmaceutique.

Le constat d'Allen Frances peut être relayé par la position de François Gonon (76), très critique vis à vis de la psychiatrie américaine qui note : "qu'aucune classification ne résoudra la question de la médicalisation excessive de la souffrance psychique » (76, p. 291). Selon nous, c'est précisément la chimio-résistance des organisations paranoïaques au traitement qui peut expliquer, Outre Atlantique, le désintérêt progressif conjugué des psychiatres placés en situation d'impuissance au niveau des prises en charge et celui des laboratoires pharmaceutiques. Ce qui montre encore davantage la nécessité de se préoccuper plutôt de la dynamique intrapsychique sous tendant le sous-bassement symptomatique plutôt que de se référer à la seule neurobiologie.

Le manque de données globales concernant ces entités lésées par le remaniement dans la nosographie psychiatrique semble faire consensus. Les auteurs s'accordent à dire que les recherches sont quasi inexistantes concernant le trouble de la personnalité paranoïaque, mais paradoxalement, certains acceptent volontiers qu'il soit supprimé. Qu'est-ce qui justifie ces remaniements? Sur quoi s'appuient ces décisions nosographiques?

\subsection{La critique de l'axe II}

Si le modèle dimensionnel DSM-5 offre, en effet, l'accès à un continuum entre le normal et le pathologique dans l'appréhension holistique de la personnalité, les experts de la nosographie psychiatrique anglo-saxonne (tels que 77,31 ) ont dénoncé de nombreuses lacunes dans l'axe II du nouveau modèle. Dès 2011, Skodol et al. (77) annonçaient que les troubles de la personnalité paranoïaque étaient destinés - au côté de trois autres troubles, schizoïde, histrionique et dépendant - à disparaitre dans le DSM-5. Cette nouvelle a créé une polémique au sein de la communauté scientifique, à l'exemple d'Hopwood et Thomas (16), qui, d'emblée, encourageaient les chercheurs à apporter les preuves empiriques de la validité des troubles de la personnalité paranoïaque, comme un syndrome autonome :

«L'identification des mécanismes spécifiques aux troubles de la personnalité paranoïaque fournirait des preuves plus convaincantes pour le reconnaître comme un syndrome d'origine naturelle et distincte. » (Ibid., p. 435). 
Le groupe de travail qui a élaboré l'axe II (Personality Disorders) du DSM-5 a souligné la nécessité de réduire la comorbidité des troubles au sein des diagnostics. Or, dans une étude qui porte sur 2150 patients de l'hôpital psychiatrique de Rhodes Island, Mark Zimmerman Iwona Chelminski, Diane Young, Kristy Dalrymple, Jennifer Martinez et Theresa A. Morgan, (78) trouvent que:

«Les résultats de la présente étude, ainsi que les résultats d'autres études, ne fournissent pas de preuves claires pour la rétention préférentielle de certains troubles de la personnalité sur les autres ». (78, p. 940)

Les résultats de Zimmerman et al., comme ceux de Skodol, invalident l'argument de l'A.P.A. pour la sélection de certains syndromes plutôt que d'autres à partir de l'intention de base réduire les facteurs de comorbidité des troubles de l'axe II.

Blais et al. (30) ont, quant à eux, interrogé le diagnostic de 280 patients et mettent à jour des données qui vont à l'encontre de la suppression de ces troubles. L'analyse factorielle révèle clairement huit facteurs significatifs (au lieu de six conservés dans le nouveau modèle des troubles de la personnalité du DSM-5). Les deux à réintégrer sont le trouble de la personnalité schizoïde et le trouble de la personnalité paranoïaque dont l'élimination a, selon ces auteurs, des conséquences négatives inattendues au niveau de la pratique clinique (30, p. 326).

Les arguments en défaveur de la décision de l'A.P.A. font quasiment l'unanimité au sein de la communauté scientifique. Mullins-Sweat et Bernstein (31) ont sollicité les membres de l'ARPD (Association for Research on Personality Disorder) et de l'ISSPD (International Society for the Study of Personality Disorders) $(n=520)$ afin de recueillir leur conception des troubles des experts au plan international. Ils ont obtenu 162 réponses afin de déterminer la sélection en vue de la suppression d'un ou plusieurs troubles de la personnalité dans DSM-5 :

- Faible utilité clinique,

- Peu de preuves de sa validité.

Les experts ont considéré que tous les troubles de la personnalité sont «valides » avec le plus grand soutien envers la personnalité antisociale et borderline. Les troubles de la personnalité paranoïaque, quant à eux, ont été considérés comme «valides » à 82,8 \% ... L'accumulation de ces contradictions et de ces constats d'échec témoignent de la disjonction locale entre approche psychodynamique et approche symptomatique, puisque dans le même temps où la nosographie américaine s'est rapprochée de l'approche dimensionnelle et donc de la nosographie psychanalytique, elle a abandonné l'analyse des mécanismes intrapsychiques qui sont des outils essentiels pour différencier les fonctionnements psychiques normaux et pathologiques $(26,15)$. Seules les nosographies à base psychodynamique parviennent à maintenir ensemble ces deux aspects. Cela se vérifie au sein du Psychodynamic Diagnostic Manual (voir 79), dont la deuxième édition révisée sera prochainement publiée, au sein duquel, à l'inverse du DSM, la place de la paranoïa n'est pas remise en cause (80).

\subsection{Le problème de la comorbidité entre le DD et le PPD}

Le choix de supprimer le PPD a créé un débat animé chez les auteurs anglo-saxons spécialistes des troubles de la personnalité dans la classification nosographique psychiatrique, notamment entre les chercheurs expérimentalistes (en laboratoire) et les chercheurs cliniciens (sur le terrain). Les données ne sont pas consensuelles. Dans leur étude de 2013 déjà citée, de Portugal et al. (44) investiguent les liens entre la symptomatologie de DD et de PPD.

La comorbidité entre DD et PPD est donc élevée ce qui tendrait à confirmer l'hypothèse selon laquelle PPD correspondrait au versant caractériel de la paranoïa quand DD correspondrait au versant délirant de la paranoïa. 
Dans cette étude de de Portugal et al. (44), une structure à quatre facteurs a été extraite, parmi lesquels celle que les auteurs ont nommé la «dimension paranoïde », un facteur qui regroupe cinq symptômes paranoïdes typiques (les délires, l'excitation, le manque de jugement, la méfiance et l'hostilité) et qui rend compte de $21,3 \%$ du total de la variance.

La dimension paranoïde est fortement élevée au sein de DD dans cette étude de de Portugal et al. (44) : la symptomatologie du paranoïaque infiltre ce trouble, comme les auteurs l'avaient prédit.

\subsection{La prévalence du PPD}

Selon le DSM-IV, la prévalence des troubles de la personnalité paranoïaque a été rapportée comme étant de $2 \%$ à $30 \%$ en milieu clinique, alors qu'en référence au DSM-IV-R, sa limite inférieure est vingt fois plus faible puisqu'elle est estimée à $0,1 \%(81)$ et sa limite supérieure un peu plus faible, évaluée à $27,6 \%$ (82) en fonction des méthodes diagnostiques utilisées et de la nature de l'échantillon étudié. Le système classificatoire dans ce contexte fait défaut puisqu'en fonction de la méthode diagnostique et donc de la sensibilité du clinicien, le PPD est considéré comme un trouble rare ou au contraire comme un trouble très fréquent. Est-ce parce que certains cliniciens ont d'ores et déjà délaissé cette entité au profit d'autres catégories diagnostiques?

Si l'on s'intéresse maintenant aux travaux scientifiques relatifs à la prise en charge des sujets présentant des troubles de la personnalité paranoïaque, il convient de tenir compte du travail de Karterud et al. (83) qui, dans une étude portant sur un traitement chimique, repèrent que le PPD est l'un des trois troubles les plus courants des patients souffrant d'un trouble de la personnalité. De même, grâce aux études relatives à la prise en charge psychiatrique, on relève que le trouble de la personnalité paranoïaque serait une classe jugée «importante » (selon la réponse de $82,8 \%$ des experts) pour la prise de décision des praticiens lors de l'instauration du traitement. Dans une enquête plus récente, Mullins-Sweat et al. (31) observent que les experts considèrent majoritairement que les personnalités schizoïdes et paranoïaques font l'objet d'une prise en charge et de traitement fréquents en dépit de la pauvreté des travaux scientifiques sur cet aspect.

Concomitamment, si l'on se fie aux travaux de Blais et al. (30), qui, dans une perspective clinique, ont étudié la proposition diagnostique chez 280 patients (se basant sur des diagnostics établis sur le terrain), via une analyse factorielle, alors on constate que le trouble de la personnalité paranoïaque apparait en troisième position (30\%), juste derrière le trouble de la personnalité borderline (56\%). Cette étude qui porte sur un nombre important de sujets semble démontrer que le PPD est un trouble plus commun que ne le laisse supposer les études précédentes. Cette recherche attire d'autant plus notre intérêt, et a d'autant plus de valeur selon nous, qu'elle est basée sur une recherche de terrain, à partir de diagnostics déjà posés.

\subsection{Conclusion intermédiaire}

Le niveau de comorbidité élevé associé au PPD, ainsi que le manque de recherches probantes sur cette entité, ont conduit les auteurs du DSM-5 modèle hybride à le supprimer comme entité distincte. Selon nous, ceci est regrettable puisque ce trouble est reconnu par les experts et les cliniciens et que c'était celui (avec le DD) qui permettait encore, au sein de la nosographie psychiatrique, de classifier la personnalité paranoïaque. Si un tel débat a eu lieu entre les auteurs anglo-saxons, spécialistes des troubles, c'est parce que cette disparition va créer un vide nosographique important réel et que les prises en charge thérapeutiques risquent de ne plus pouvoir être adaptées.

L'interprétation qui a été faite des données épidémiologiques sur les traits de personnalité paranoïaques tend à confondre un mécanisme de défense (la projection) potentiellement 
observable chez tout un chacun et la paranoïa en tant que structure de la personnalité. Comme Catherine Azoulay (53, p. 37) l'écrit :

«Dans le champ de la clinique du sujet tout-venant, on peut considérer que chacun de nous possède son germe de paranoïa dans les relations sociales, professionnelles, familiales, par une référence au regard de l'autre sur soi qui renvoie, par retour du projeté, ce qu'on ne veut penser sur soi-même. En ce sens, la projection est au fondement même de toute attribution inconsciente de contenus psychiques à l'extérieur. »

Dans une perspective psychodynamique post-freudienne le mouvement projectif chez le sujet tout venant consiste à lutter contre les affects de déplaisir par une projection à l'extérieur tandis que chez le sujet paranoïaque, il s'agit de lutter contre des désirs homosexuels et par «ce qui devrait être ressenti intérieurement comme de l'amour est perçu extérieurement comme de la haine ». (41, p. 311).

Si la psychiatrie actuelle n'a pas pris en compte cette distinction entre la paranoïa comme défense (paranoïa-trait) et la paranoïa structurelle, c'est certainement parce qu'elle ne s'intéresse pas à la dynamique intrapsychique qui organise le fonctionnement de la personnalité. Selon le modèle freudien, il n'est pas étonnant de retrouver des traits de personnalité paranoïdes chez les sujets « sains » puisque tout à chacun est amené à utiliser ce mécanisme de défense (la projection). La différence entre le paranoïaque et le sujet toutvenant, c'est que le premier l'utilise de façon massive et contre une angoisse de morcellement patente. Ce que les épidémiologistes ont mis en lumière n'est ni plus ni moins que ce que les auteurs post-freudiens reconnaissent depuis des décennies déjà. Or, ce constat établi, les auteurs du DSM-5 ont pris le parti de supprimer un trouble (qui correspond au caractère paranoïaque) de leur nouveau modèle comme si ce dernier ne pouvait plus être structurel à partir du moment où il est présent comme une défense adaptative chez une grande partie de la population. En interprétant les données quantitatives de la sorte, les adeptes d'une classification sur des bases symptomatologiques ont révélé les limites de leur méthode.

Une classification basée uniquement sur la symptomatologie ne permet pas de distinguer la paranoïa structurelle de sa fonction défensive comme dans les symptômes d'hypervigilance du syndrome de stress post-traumatique associés au fait de « se sentir constamment menacé ». Les symptômes sont très semblables, et même peut être indiscernables avec ceux de la paranoïa. Parmi les troubles de la personnalité, les troubles de la personnalité schizotypiques comprennent également des éléments relatifs à la paranoïa parmi ses critères, et les symptômes du trouble borderline de la personnalité DSM-IV comprennent aussi des phénomènes «transitoires liés au stress de l'idéation paranoïaque ». Or, la compétitivité excessive d'un patient présentant un trouble de la personnalité narcissique se réfléchit par rapport aux failles narcissiques que présentent ces personnalités. Ces éléments convergent tous en défaveur d'une classification symptomatologique des troubles, pourtant les auteurs du DSM persévèrent dans cette direction, préférant ignorer les failles de leur système (trop superficiel) à défaut de l'adapter et en dépit des critiques des différents auteurs que nous avons évoqués. La paranoïa serait donc « victime » d'un tel système nosographique.

\section{Diagnostic différentiel entre paranoïa et schizophrénie}

Les changements opérés pour la classification de la paranoïa sont concomitants à d'autres variations des catégories voisines. Les inclure dans cette revue de littérature donne une idée de la façon dont le tableau paranoïaque sera diagnostiqué avec ce nouveau modèle. Nous allons nous concentrer sur la principale révolution opérée qui est la mise en place d'un chapitre global sur les «troubles du spectre de la schizophrénie et autres troubles psychotiques ». Puis, nous discuterons d'études portant sur certains éléments différentiels 
entre paranoïa et schizophrénie associés aux troubles de la pensée, à la symptomatologie délirante, à l'adaptation psychosociale, au pronostic et à la valence paranoïde.

\subsection{L'évolution de la schizophrénie dans le DSM}

La définition de la schizophrénie a évolué au cours des six éditions du DSM. Trois axes majeurs ont été empruntés pour la définir :

- la définition de Kraepelin (12) qui met l'accent sur l'évolution, la chronicité et les perspectives évolutives pauvres ;

- la définition de Kahlbaum (84) de l'hébéphrénie (qui apparaît au moment de l'évolution pubérale et qui se termine par la démence). Kahlbaum isole une forme pseudo-psychopathique atténuée voire curable: l'héboïdophrénie. Son nom est également attaché à la catatonie à laquelle il consacre une remarquable monographie en 1874 ;

- la définition bleulérienne (85) avec l'accent mis sur la pathologie dissociative en tant qu'élément fondamental et en second lieu les symptômes négatifs ;

- les descriptions psychopathologiques de Schneider (27) sur les symptômes positifs (par exemple, la distorsion de la réalité) et les symptômes négatifs.

L'importance donnée à ces quatre perspectives a toujours varié dans le temps. Comme le notent Random Tandon et al. (86), la perspective de Bleuler a davantage imprégné le DSM-I et le DSM-II ce qui a conduit à un écart important entre les marqueurs symptomatologiques utilisés par les américains et ceux utilisés en Grande-Bretagne et en Europe plus globalement. Le DSM-I était également influencé par la notion de « réaction » schizophrénique d'Adolf Meyer; le DSM-II par une définition large autour de la perte des frontières du moi. Ce serait dans un élan consensuel, en réaction à «ces inconsistances », que les auteurs du DSM-III auraient adopté des critères plus exigeants parmi lesquels la chronicité et les défaillances cognitives fonctionnelles ainsi que la liste des symptômes de premier rang de Schneider (86). Depuis le DSM-III en passant par le DSM-III-R jusqu'au DSM-IV, les modestes modifications des critères de la schizophrénie ont quant à elles concerné l'âge limite d'apparition de la maladie, auparavant fixé à 45 ans, ainsi que l'inclusion de la symptomatologie négative.

Récemment, le trouble délirant a été intégré au sein du «spectre schizophrénique » dans le DSM-5 (23), qui comprend la schizophrénie, d'autres troubles psychotiques et le trouble schizotypique de la personnalité. Ces entités sont définies par des anomalies dans un ou plusieurs domaines symptomatiques parmi les cinq suivants :

1) les délires, définis par les auteurs selon la proposition suivante :

«Des croyances qui ne sont pas susceptibles de changer à la lumière des éléments de preuve contradictoires fixés. Leur contenu peut inclure une variété de thèmes (par exemple, de persécution, référentielle, somatique, religieux, grandiose). » (23, p. 126).

2) les hallucinations, qui correspondent dans le DSM-5 à :

«Des expériences de perception qui se produisent sans stimulus externe. Ils sont vifs et clairs, avec toute la force et l'impact des perceptions normales, et incontrôlables ». (23, p. 126)

3) la désorganisation de la pensée, définie par la proposition suivante :

«La désorganisation de la pensée (de trouble de la pensée formelle) est généralement repérée chez la personne à travers son discours. L'individu peut passer d'un sujet à l'autre (déraillement ou associations libres). » (23, p. 126) Le symptôme doit être sévère.

4) la désorganisation psychomotrice (dont la catatonie) :

«Peut se manifester de diverses façons, allant de la "bêtise enfantine" à l'agitation imprévisible. Des problèmes peuvent être notés dans toute forme de comportement orienté 
vers un but, conduisant à des difficultés dans l'exécution des activités de la vie quotidienne. » (23, p.126)

5) les symptômes négatifs qui correspondent à (i) la diminution de l'expression émotionnelle et à la perte de la motivation; (ii) l'alogie, l'anhédonie et le retrait social. Les deux premiers symptômes négatifs étant les plus importants pour poser le diagnostic.

Plus précisément, le diagnostic différentiel entre trouble délirant (DD), d'une part, et schizophrénie et trouble schizophréniforme, d'autre part, se fait à partir de l'absence des autres symptômes caractéristiques de la phase active de la schizophrénie (23, p. 93).

Un tel regroupement établit un continuum entre les tableaux schizophréniques, paranoïaques et paraphréniques, luttant contre l'éparpillement des catégories de la psychose. Toutefois, il est opéré à partir d'assimilations approximatives entre des symptômes et aucunement par le repérage d'une logique de défense psychotique qui serait à l'œuvre au-delà de ces catégories (49).

\subsection{Les modifications entre DSM-IV et DSM-5}

Deux changements ont été faits pour le critère A de schizophrénie dans le DSM-5 :

\section{Critère A (A.P.A., 2013)}

A. Deux (ou plus) parmi les critères suivants, chacun devant être présent dans une proportion significative du temps au cours d'une période d'un mois (ou moins en cas de traitement efficace). Au moins un parmi les critères (1), (2) ou (3) doit être présent.

1. Idées délirantes

2. Hallucinations

3. Discours désorganisé (incohérences ou déraillements fréquents)

4. Comportement grossièrement désorganisé ou catatonique

5. Symptômes négatifs (aboulie ou diminution de l'expression émotionnelle).

Les auteurs ont procédé à l'élimination de l'attribution spéciale des hallucinations auditives que Schneider (27) classifiait de «premier rang » (c'est-à-dire entendre deux ou plusieurs voix converser), ce qui reflète l'impact des recherches sur l'entente de voix (87).

Le spectre schizophrénique s'élargit encore davantage et tend à " absorber » l'ensemble des troubles psychotiques dans un mouvement d'indifférenciation. C'est ainsi que le sous-type paranoïde a également disparu. Le constat établi par les auteurs tel que Blais et al. (30) et Mullins-Sweat et al. (31) sur la perte en matière de prise en charge est donc particulièrement d'actualité. Quels sont les risques à traiter un patient souffrant de paranoïa comme s'il s'agissait d'une schizophrénie ? Nous allons tenter de répondre à cette question à travers les points suivants.

\subsection{La pensée paranoïde en tant que prédicteur d'une entrée dans la psychose?}

Dans une perspective dimensionnelle des troubles Wilcox, Briones, Quadri et Tsuang (61) ont mené une étude portant sur une population de 174 jeunes patients adultes. Ces auteurs (61) montrent qu'évaluer des traits de personnalité paranoïaque (via l'échelle de paranoïa de Fenigstein, 1992) et des troubles de la pensée au sein de l'examen clinique permet d'améliorer la prédiction de la conversion psychotique et fournit des résultats à long terme. 
Selon les observations de Wilcox et al. (61), l'apparition précoce d'idées de référence ainsi que la pauvreté de la pensée semblent être des prédicteurs significatifs de la détérioration future. La «pensée paranoïde » est dès lors considérée comme un facteur de risque de développement de la schizophrénie.

Mais peut-on isoler la pensée paranoïde et à quoi renvoie-t-elle ?

\subsection{La paranoïa versus le spectre schizophrénique, une entité distincte ?}

\subsection{Facteurs de comorbidité entre le trouble délirant et le spectre schizophrénique}

Serreti et al. (39) ont réalisé une analyse factorielle sur 100 patients diagnostiqués de schizophrénie paranoïde afin de déterminer la symptomatologie qui appartenait aux troubles délirants, les auteurs relèvent que cette dernière est présente chez la plupart des sujets (la certitude de la véracité des constructions délirantes) et que les comportements relatifs au trouble délirant sont présents également (engagement, excentricité et leur influence sur le comportement).

L'étude de Dilip V. Jeste et Alice J Krull (88) qui a porté sur un échantillon de 17 patients avec trouble délirant versus 12 patients schizophrènes paranoïdes valide l'entité «trouble délirant» dans la majorité des cas (sur la base des données cliniques et de tests neuropsychologiques) mais met en évidence que, dans une minorité des cas, le trouble délirant dépendrait d'un développement schizophrénique. De sorte que si on trouve les symptômes du trouble délirant au sein de la schizophrénie, le contraire (symptomatologie schizophrénique au sein du trouble délirant) n'est pas valable.

Dans une recherche récente portant sur portant sur 135 sujets $\mathrm{DD}, 373$ sujets schizophrènes et 40 sujets diagnostiqués de troubles schizo-affectifs, le trouble délirant émerge comme une entité psychopathologique distincte plus bénigne que la schizophrénie et le trouble schizoaffectif (90).

Ces observations militent, à notre avis, en faveur de la nécessité de séparer le DD du spectre schizophrénique et de le réintégrer comme un marqueur du fonctionnement paranoïaque...

\subsection{Eléments différentiels : I'adaptation psychosociale et le pronostic}

Harold I. Kaplan et Benjamin J. Sadock (90) soulignent que les paranoïaques sont des personnes qui peuvent être en mesure de s'adapter en dehors de l'hôpital, même si elles souffrent d'une évidente symptomatologie. Le paranoïaque s'adapte mieux en société que d'autres psychotiques car les idées et les comportements paranoïdes causent généralement moins de perturbations, et car, par endroits, la paranoïa s'avère être une réponse appropriée aux dangers réels de la vie en dehors de l'hôpital.

D'autre part, dans les hôpitaux, les paranoïaques se trouvent généralement en difficulté avec les règles de l'institution et avec le comportement intrusif du personnel et des autres patients. En conséquence, ils ne s'adaptent pas bien à ce milieu et souhaitent mettre fin à leur hospitalisation le plus tôt possible. Le personnel soignant de son côté supporte mal la présence, dans son service, de ce genre de patients considérés comme «non coopératifs ».

Quant au pronostic, pour les auteurs germaniques, à l'instar de Karl Leonhard (91), ainsi que pour Barry Ritzler (92) et pour Jill M.Goldstein (93) celui de la paranoïa serait meilleur que pour les autres états psychotiques. Daniel S. Papernick, Herbert Pardes et Arnold Winston (94) ont également constaté que les patients schizo-paranoïdes hospitalisés qui présentaient des délires grandioses montraient moins de possibilités d'amélioration que les patients paranoïaques. 


\subsection{La schizophrénie paranoïde}

La valence paranoïde, au sein d'une pathologie schizophrénique, présenterait un facteur pronostique également plutôt favorable. Les patients schizophrènes non paranoïdes se situeraient à un niveau plus bas d'adaptation que les schizophrènes paranoïdes selon Ritzler (92). Ces mêmes auteurs considèrent aussi que dans la schizophrénie, la perte du délire paranoïaque résulterait d'un signe de détérioration chronique plutôt que de l'amélioration dans le fonctionnement psychologique du patient (92, p. 713). Au regard de la psychopathologie psychodynamique cette observation est cohérente avec le fait établi par de nombreux auteurs qu'un noyau paranoïaque dans la schizophrénie permettrait au sujet de lutter contre la fusion totale avec la mère $(95)$.

\section{Conclusion}

A travers cette revue de la littérature, nous avons tenté de cibler le débat actuel au sujet de la paranoïa et de sa disparition dans la nosographie américaine au sein de la communauté scientifique internationale. Nous constatons :

(i) une absence de consensus, avec des apports empiriques insuffisants pour trancher ;

(ii) un impact négatif de cette carence pour la prise en charge des patients: l'inadéquation entre le tableau clinique et la nosographie rend plus difficile l'accès au système de soin ;

(iii) un problème méthodologique avec des modes d'évaluations inadaptées (autoquestionnaires sans échelle de contrôle).

Les révisions de la classification américaine de la paranoïa ne nous semblent pas reposer sur des arguments cliniques valables. Il ne s'agit pas que d'une résistance face à la perte d'habitudes de penser, mais bien du constat d'un étiolement de la finesse clinique dans le repérage et le diagnostic différentiel de la paranoïa. La montée en puissance du modèle dimensionnel n'a pas eu les conséquences attendues, puisque le DSM-5 l'interprète uniquement selon une approche quasi-dimensionnelle, c'est-à-dire en répartissant les «catégories» de symptômes dans des «dimensions» d'expression. A ce titre, il n'opère qu'une extension du champ de la maladie mentale qui fait le jeu des industries pharmaceutiques. Il n'est nullement question dans ce cadre d'intégrer les apports de la psychologie ou de la psychanalyse, ce qui reviendrait à croiser cet axe psychopathologique avec un axe de personnalité venant le moduler. L'approche pleinement dimensionnelle, qui est par exemple celle de la clinique psychanalytique structurale, est plus à même de rendre compte des formes de résilience ou des combinaisons de symptômes avec des fonctionnements psychiques de qualité $(26,80)$.

Or, la paranoïa est exemplaire de ce problème puisqu'elle «appartient à la fois à la psychologie et à la psychopathologie » $(96$, p. 102). Sa symptomatologie de surface est depuis longtemps examinée en prenant en compte son pendant dans le caractère. Si l'on se place dans une perspective structurale, lorsque l'on s'intéresse au sous-bassement psychique d'un trouble, on peut inscrire la structure paranoïaque au sein de la lignée structurelle des psychoses dissociées tout en admettant que son expression psychopathologique peut prendre des formes diverses... y compris une symptomatologie très discrète dans le cas des formes compensées. Il faudrait même poser une distinction fondamentale entre la logique paranoïaque, capable d'affecter des individus autrement indemnes de troubles mentaux, et le registre de la psychose (97). Sans aller jusqu'à dire que tous les sujets « sains » présentant des traits paranoïdes s'inscrivent (ou non) dans une structure de personnalité paranoïaque, cette approche aurait pu permettre de créer l'ébauche d'un consensus entre la psychiatrie et la psychopathologie psychanalytique, l'une basée sur le symptôme de surface et la seconde sur 
les mécanismes intrapsychiques sous-tendant ces symptômes. La perspective pleinement dimensionnelle aurait permis de conserver la paranoïa à la fois comme une entité indépendante et comme une structure articulée aux autres formes de logique défensive de la psychose. Le choix des auteurs du DSM-5 a été plus simple et plus radical puisqu'ils ont supprimé ce qu'il restait de la paranoïa dans le modèle hybride, quasi-dimensionnel et innovateur du DSM-5.

Comme nous l'avons vu, le parti pris de la nosographie américaine consistant à s'appuyer uniquement sur le symptôme pour classifier les troubles a ainsi créé la confusion au sein de la communauté scientifique, incapable actuellement de s'accorder sur la question de la classification de la paranoïa. Dès lors, nous sommes en droit de nous demander, sans tomber nous-mêmes dans un imaginaire paranoïde : à qui profitent ces révisions ?

\section{Références}

(1) Borch-Jacobsen M. La fabrique des folies. De la psychanalyse au psychopharmaketing. Paris : Sciences humaines, 2013.

(2) Maleval JC. Eléments pour une appréhension clinique de la psychose ordinaire: Séminaire de la découverte freudienne, Rennes, 18-19 janvier 2003

(3) Kirk S, Kutchins H. Aimez-vous le DSM ? Le triomphe de la psychiatrie américaine. Paris : Institut Synthélabo, 1998.

(4) Frances A. Sommes-nous tous des malades mentaux ? Le normal et le pathologique. Paris : Odile Jacob ; 2013.

(5) Le Moigne P. Une nouvelle carte du normal et du pathologique. L'institutionnalisation de la santé mentale aux Etats-Unis. Sciences sociales et santé 2010 ; 28(1) : 81-108.

(6) Minard M. Le DSM-roi. La psychiatrie américaine et la fabrique des diagnostics. Paris : Erès ; 2013.

(7) Watters E. Crazy like us: The Globalization of the American Psyche. New York : Free Press ; 2010.

(8) Garrabé J. Nosographie et classifications dans l'histoire de la psychiatrie. Evol Psychiatr 2014; 79(1): 5-18.

(9) Sander W. Ueber eine specielle Form der primären Verrücktheit. Archiv für Psychatrie und Nervenkrankheit $1868 ; 1: 387-419$.

(10) Snell L. Ueber Monomanie als primäre Form der Seelenstörung. Allgemeine

Zeitschrift für Psychiatrie $1865 ; 22: 368-381$.

(11) Krafft-Ebing R. Psychopathia Sexualis. Paris : Georges Carré ; 1888.

(12) Kraepelin E. Psychiatrie, Ein Lehrbuch für Studirende und Aerzte, 6. Leipzig : Auflage ; 1899.

(13) Lévy B. Die Paranoiafrage. Mémoire de Master 2 (non publié) - Université de Paris VII , 2012.

(14) Castel R. La gestion des risques. De l'anti-psychiatrie à l'après-psychanalyse. Paris :

Les Editions de Minuit, 2011 ( $2^{\mathrm{e}}$ édition).

(15) XXX 2015

(16) Hopwood CJ, Thomas KM Paranoid and Schizoid Personality Disorders. In : Widiger $\mathrm{T}$, editors. The Oxford Handbooks of Personality Disorders. Oxford : Oxford library of psychology ; 2012.

(17) Kraepelin E. Introduction à la psychiatrie clinique. Paris : Navarin, 1984.

(18) Serieux P, Capgras J. Les folies raisonnantes. Paris : Alcan, 1909.

(19) Bleuler E. Lehrbuch der Psychiatrie. Berlin : Springer, 1916.

(20) American Psychiatric Association. DSM III, Diagnostic and Statistical Manual of 
Mental Disorders, 3rd Edition, American Psychiatric Association. Washington: DC; 1980.

(21) American Psychiatric Association. DSM-IV-TR, Diagnostic and Statistical Manual of Mental Disorders, 4th Edition, Text Revision, American Psychiatric Association. Washington : D.C ; 1994.

(22) American Psychiatric Association. DSM-IV-TR, Diagnostic and Statistical Manual of Mental Disorders, 4th Edition, Text Revision, American Psychiatric Association. Washington : D.C ; 2000.

(23) American Psychiatric Association et DSM-5 Task Force, Diagnostic and Statistical Manual of Mental Disorders: DSM-5. American Psychiatric Association. Washington : D.C.; 2013.

(24) Levy B. Genèse, évolution et pertinence de la notion de délires paranoïaques : approche épistémologique, culturelle et clinique. Paris 7 (thèse non soutenue)

(25) Racamier PC. Esquisse d'une clinique psychanalytique de la paranoïa. Revue française de psychanalyse $1966 ; 30(1): 145-72$.

(26) Bergeret J. La personnalité normale et pathologique (3e éd.). Paris: Dunod: 2013.

(27) Schneider K. Les personnalités psychopathiques. Paris: PUF; 1955.

(28) Evrard R. Folie et Paranormal : vers une clinique des expériences exceptionnelles. Rennes : PUR ; 2014.

(29) Kenberg O. Les troubles graves de la personnalité. Paris : PUF ; 2004

(30) Blais MA, Malone JC. Structure of the DSM-IV Personality disorders as revealed in clinician ratings. Comprehensive Psychiatry 2013 ; 54, 326-33

(31) Mullins-Sweatt SN, Bernstein DP, Widiger TA. Retention or deletion of personality disorder diagnoses for DSM-5 : An expert consensus approach. Journal of personality disorders $2012 ; 26(5): 689-703$.

(32) Organisation Mondiale de la Santé. CIM-10, Critères diagnostiques pour la recherché et la classification international des maladies. Paris: Masson; 1994.

(33) McWilliams N. Psychoanalytic Diagnosis: Understanding Personality Structure in the Clinical Process. Guilford Press ; 2011 a.

(34) Skodol AE. Personality disorders in DSM-5. Annual Review of Clinical Psychology $2012 ; 8: 317-344$.

(35) Maâlej M, Ben Mahamoud S, Fki H, Zouari L, Rakam A, Zouari N, Damak J. Approche épidémiologique du trouble délirant, à propos de 66 cas. Annales MédicoPsychologiques 2006 ; 164: 388-394

(36) Tiernan B, Tracey R, Shannon C. Paranoia and self-concepts in psychosis : A systematic review of the literature. Psychiatry Research 2014; 216 : 303-313.

(37) Fear CF, MacMonagle T, Healy D. Delusional disorders : boundaries of a concept. Eur Psychiatry $1998 ; 13: 210-8$.

(38) Munro A. Delusional disorder. Cambridge : University Press; 1999.

(39) Serretti A, Lattuada E, Cusin C, Smeraldi E. Factor analysis of delusional disorder symptomatology. Comprehensive Psychiatry 1999; (40(2): 143-147.

(40) Freud S. Remarques psychanalytiques sur l'autobiographie d'un cas de paranoïa. In Freud S, editors. Cinq psychanalyses. Paris : PUF, 1954.

(41) De Tychey. Le Rorschach en clinique de la dépression adulte. Paris : Dunod, 2012.

(42) Lacan J. Le séminaire, Livre III, Les psychoses. Paris : Éd. Du Seuil ; 1981.

(43) Winokur G, Morrison J, Clancy J, Crowe R. Iowa 500: The clinical and genetic distinction of hebephrenic and paranoid schizophrenia. Journal of Nervous and Mental Disease 1974 159:12-19

(44) De Portugal E, Diaz-Caneja M, Gonzalez-Molinier M, de Castro MJ, del Amo V, 
Arango C, Cervilla JA. Prevalence of premorbid personality disorder and its clinical correlates in patient with delusional disorder. Psychiatry research 2013; 210(3): 986-993.

(45) Berenbaum H, Fujita F. Schizophrenia and personality: Exploring the boundaries and connections between vulnerability and outcome. Journal of Abnormal Psychology 1994 ; $103: 148-158$

(46) Madeline M, Gladis D, Levinson F, Mowry J. Delusions in schizophrenia spectrum disorders : diagnostic issues. Schizophrenia Bulletin 1994 ; 20 (4).

(47) Mojtabai R, Nicholson RA. Inter rater reliability of ratings of delusions and bizarre delusions. Am. J. Psychiatry 1995 ; 152 :1804-1806.

(48) Bottéro A. Remettre en cause les critères DSM IV de la schizophrénie. Neuropsychiatrie : tendances et débats $2000 ; 8: 43-44$.

(49) Maleval JC. La logique du délire. Rennes : PUR ; 2011.

(50) Kendler KS, Glazer WM, Morgenstern H. Dimensions of delusional experience. Am.J. Psychiat. $1983 ; 140$ : 466-469.

(51) Madeira LA, Manarte LF, Guerreiro D, Dias C. A case of delusional disorder, diagnostics and therapeutic questions. European psychiatry 2011; $26: 1709$.

(52) Suvisaari J, Perälä J, Samuli I, Härkänen T, Pirkola S, Joukamaa M, Koskinen S, Lönnqvist J, Reunanen A. Type 2 diabetes among persons with schizophrenia and other psychotic disorders in a general population survey.Psychiatry and Clinical Neuroscience 2008 ; 258(3) : 129-136.

(53) Azoulay C. Les mécanismes paranoïaques dans les épreuves projectives : revue des principaux auteurs. Psychologie clinique et projective 2007 ; 13(1) : 33-56.

(54) Hempel CG. Aspects of scientific explanation. New-York :Free Press ; 1965.

(55) Eysenck HJ. A model for personality. New York: Springer-Verlag ; 1981.

(56) Svrakic DM, Whitehead C, Przybeck TR, Cloninger CR. Differential diagnosis of personality disorders by the Seven-Factor Model of Temperament and Character. Archieves General of Psychiatry 1993; 50: 991-999.

(57) Saulsman LM, Page AC. The five-factor model and personality disorder empirical literature: A meta-analytic review. Clinical Psychology Review $2001 ; 23: 1055-1085$.

(58) Bagby RM, Costa PT, Widiger TA, Ryder AG, Marshall M. DSM-IV Personality Disorders and the Five-Factor Model of Personality: A Multi-Method Examination of Domain- and Facet-Level Predictions. European Journal of personality 2005 ; 19 : 307324.

(59) Daneluzzo E, Stratta P, Rossi A. The contribution of temperament and character to schizotypy multidimensionality. Comp. Psychiatry 2005; 46:50-55.

(60) Freeman D. Suspicious minds : The psychology of persecutory delusions. Clinical Psychology Review 2007 ; 27 :425-457.

(61) Wilcox J, Briones D, Quadri S, Tsuang M. Pronostic implications of paranoia and thought disorder in new onset psychosis. Comprehensive psychiatry $2014 ; 55: 813-817$.

(62) Bricaud M, Calvet B, Viéban F, Prado-Jean A, Clément JP. Étude Per Cadim : relations entre approches dimensionnelles et catégorielles de la personnalité. L'éncéphale 2011 ; 38 :288-295.

(63) Kretschmer E. Paranoïa et sensibilité. Paris : PUF ; 1963.

(64) Gurpegui M, Jurado D, Fernandez-Molina MC, Moreno-Abril O, Luna JD, Alarcon RD. Personality profiles and minor affective psychopathology in a non clinical sample: An empirical verification of Cloninger's theoretical model. Journal of Affective Disorders 2009; $119: 34-42$.

(65) Chan R, Xiaoyan L, Lai MK, Li H, Wang Y, Cui J, Deng Y, Raine A. Exploratory study on the base-rate of paranoid ideation in a non-clinical Chinese sample. Psychiatry Research 2011; $185: 254-260$. 
(66) Torgersen S, Kringlen E, Cramer V. The prevalence of personality disorders in a community sample. Archives of General Psychiatry 2001 ; 58(6) : 590-596.

(67) Triebwasser J, Chemerinski E, Roussos P, Siever LJ. Paranoid Personality Disorder. Journal of Personality Disorders $2012 ; 26$ :055.

(68) Coid, J., Yang, M., (2006). Prevalence and correlates of personality disorder in Great Britain. The British Journal of Psychiatry, 188, 423-431.

(69) XXX, 2015

(70) Huprich SK. Rorschach assessment of the personality disorders. Mahwa: N.J. Erlbaum, 2006.

(71) Deloro C. Caractère, constitution, structure. Lacan et le premier concept de paranoïa. L'Évolution Psychiatrique $2011 ; 75,391-401$.

(72) Bernstein DP, Iscan C, Maser J. Opinions of personality disorder experts regarding the DSM-IV personality disorders classification system. Journal of Personality Disorders 2007, 21, 536-551

(73) Birkeland SF. Paranoid Personality Disorder and the schizophrenia spectrum-Where to draw the line? : Complex Paranoid Personality case. Personality and Mental Health, $2013 ; 7(3): 254-258$.

(74) Connolly AJ, Cobb-Richardson P, Ball SA. Personality disorders in homeless dropin center clients. Journal of Personality Disorders 2008; 22(6): 573-588.

(75) Kelly BD, Casey P, Dunn G, Ayuso- Mateos JL, Dowrick, C. The role of personality disorder in "difficult to reach" patients with depression: Findings from the ODIN study. European Psychiatry 2007 ; 22(3) : 153-159.

(76) Gonon F. Quel avenir pour les classifications des maladies mentales? Une synthèse des critiques anglo-saxonnes les plus récentes. L'information psychiatrique 2013; 89: 285 94.

(77) Skodol AE, Bender DS, Morey LC, Clark LA, Oldham JM, Alarcon RD, Siever LJ. (2011). Personality disorder types proposed for DSM-5. Journal of Personality Disorders $2011 ; 25(2): 136-169$.

(78) Zimmerman M, Chelminski I, Young D, Dalrymple K, Martinez J, Morgan, TA. Which DSM-IV personality disorders are most strongly associated with indices of psychosocial morbidity in psychiatric outpatient ? Comprehensive Psychiatry $2012 ; 53$ : 940-945.

(79) Bouvet C. Le Psychodynamic Manual Diagnostic (PDM) améliore-t-il le Diagnostic and Statistical Manual IV-R (DSM-IV-R) pour la classification des pathologies des adultes? L'exemple des troubles psychotiques et des personnalités schizoïdes et paranoïdes. L'Evolution Psychiatrique 2010, 75 (4), 655-658.

(80) Huprich SK, McWilliams N, Lingiardi V, Bornstein RF, Gazzillo F, Gordon RM. The Psychodynamic Diagnostic Manual (PDM) and the PDM-2: Opportunities to Significantly Affect the Profession. Psychoanalytic Inquiry 2015; 35(sup 1): 60-73.

(81) Kantojarvi L, Veijola J, Läksy K, Jokelainen J, Herva A, Karvonen JT, Kokkonen P, Järvelin MR, Joukamaa M. Comparison of hospital-treated personality disorders and personality disorders in a general population sample. Nordic Journal of Psychiatry 2004 ; 58(5) : 357-62.

(82) Marinangeli MG, Butti G, Scinto A, Di Cicco L, Petruzzi C, Daneluzzo E, Rossi A. Patterns of comorbidity among DSM-III-R personality disorders. Psychopathology 2000 ; 33:69-74.

(83) Karterud S, Pedersen G, Bjordal E, Brabrand J, Friis S, Haaseth O, Havaaldsen G, Irion $\mathrm{T}$, Leirvâg $\mathrm{H}$, Torum E, Urnes $\mathrm{O}$. Day treatment of patients with personality disorders: Experiences from a Norwegian treatment research network. Journal of Personality Disorders 2003 ; 17(3) : 243-262. 
(84) Kahlbaum K. Psychische Krankheinten. Berlin: von A. Hireshwald; 1874.

(85) Bleuler E. Dementia Praecox or the Group of Schizophrenias. New York: International Universities Press; 1950.

(86) Tandon, R., Gaebel, W., Barch, M., D., Bustillo, J., Gur, R., E., Heckers, D., Malaspina, D., Owen, M.-J., Schultz, S., Tsuang, M., Van Os, J., Carpenter, W. Definition and description of schizophrenia in the DSM-5. Schizophrenia Research 2013; 150: 3-10. (87) Evrard R, Le Maléfan P. Que changent les entendeurs de voix à l'écoute des hallucinations ? I : Genèse d'un mouvement, Annales médico-psychologiques 2013 ; 71(9) : 623-628

(88) Jeste D, Krull AJ. Behavioral problems associated with dementia : Diagnosis and treatment. Geriatrics 1991 ; 46(11) : 28-34.

(89) Munoz-Negro J, de Portugal E, Ibanez-Casas I, Ochoa S, Dolz M, Haro MJ, RuizVeguilla M, Luna del Castillo J, Cervilla J. A dimensional comparison between delusional disorder, schizophrenia and schizoaffective disorder. Schizophrenia Research 2015 (In press).

(90) Kaplan IH, Sadock BJ. Comprehensive group psychotherapy. Baltimore, Williams \& Wilkins ; 1971.

(91) Leonhard, K. Pronosis of paranoid states in relation to the clinical features. Acta Psychiatrica Scandinavica 1975, 51: 134-151

(92) Ritzler BA. Paranoia-pronosis and treatment:A review. Schizophrenia Bulletin $1981 ; 7$ (4) : 710-28.

(93) Goldstein M. Further data concerning the relation between premorbid adjustement and paranoid ymptomatology. Schizophrenia Bulletin 1978; 4: 236-241.

(94) Papernik DS, Pardes H, Winston A. A study of hospitalized paranoid schizophrenic with grandiose symptomatology. Psychiatry Services 1975; 26: 87-90.

(95) Dreyfus A, Husain O, Rousselle Gay Crosier I. Schizophrénie simple et schizophrénie paranoïde à travers le Rorschach: Étude comparative. Psychologie médicale $1989 ; 21$ (7), 831-841.

(96) Hanus M, Louis O. Psychiatrie pour l'étudiant. Malone: Paris; 2010

(97) Englebert J. L'herméneutique paranoïaque. Evolution Psychiatrique 2013 ; 78(2): 267-277. 